\title{
A case with acute idiopathic blue finger
}

\author{
Sibel Berksoy Hayta, Rukiye Guner
}

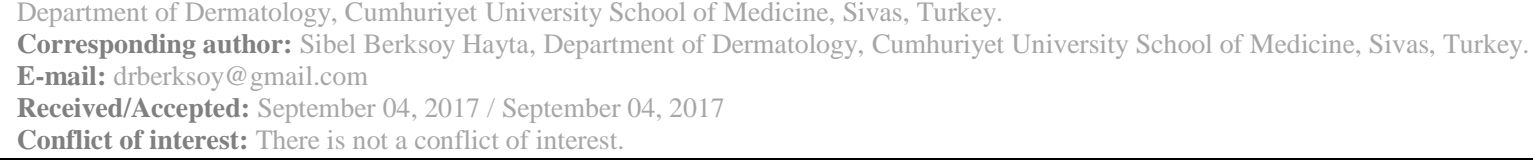

\section{To the Editor}

The acute blue finger is an uncommon disorder. It is an isolated problem and may have many causes ${ }^{1}$. The causes of isolated acute blue discoloration of a finger may be acute ischemia or vasospasm and underlying systemic illnesses. However, in some cases, there is no association with any dangerous causes or systemic diseases ${ }^{2}$. The most common etiological causes are Raynaud's disease or syndrome, infective endocarditis, peripheral vascular disease, thoracic outlet obstruction, subclavian artery stenosis, cryoglobulinemia, frostbite, trauma, vibration- induced injury and certain poisons ${ }^{1,3}$.

A 39-year-old female patient admitted with bluish discoloration of the distal phalange in the right thumb. She has no history of trauma, cold exposition, weight loss and other constitutional symptoms. She experienced two episodes of acute finger discoloration over one month. The first episode involved a spontaneous and sting purple "lump" at the base of the of the distal phalanx of her right thumb. The lesion resolved within several days spontaneously. A second episode with the same clinical symptoms involving the same finger was three weeks later after the first episode. Symptoms resolved within one day spontaneously.

Personal medical history was normal. She was physically normal in weight. She has no fever or other systemic symptoms. The blood pressure of her upper extremity was 100/60 $\mathrm{mm} \mathrm{Hg}$, symmetrically. Pulse of upper and lower extremities was normal and regular. There was no murmurs or bruits. She had no neurological sign or symptoms. There were no differences in the arterial pressure between the right and left upper limbs. Allen's test measuring arterial competency and Adson maneuver for thoracic outlet syndrome was normal. There were no embolic phenomena of the upper and lower extremities.

Local examination of right hand revealed bluish discoloration of the distal phalanx of her right thumb with sparing of tips (Figure 1). There was no swelling, tenderness, temperature variation, deformities or signs of trauma. Cold and warm exposure did not show any changes in color. Complete blood count including coagulation tests, renal and liver functions was normal. Electrocardiogram, echocardiography and chest Xray have no abnormalities. Erythrocyte sedimentation rate was $14 \mathrm{~mm} / \mathrm{h}$ and C-reactive protein $1.5 \mathrm{mg} / \mathrm{L}$. Antinuclear antibody was positive at 1:40 and negative at 1:160. Rheumatoid factor, anti-cyclic citrullinated protein, and antiSCL 70 were negative. Doppler study of her right upper limb vessels and ankle brachial index were also normal. She was ultimately diagnosed with an acute benign blue finger. She was also symptomfree for the past one month. 


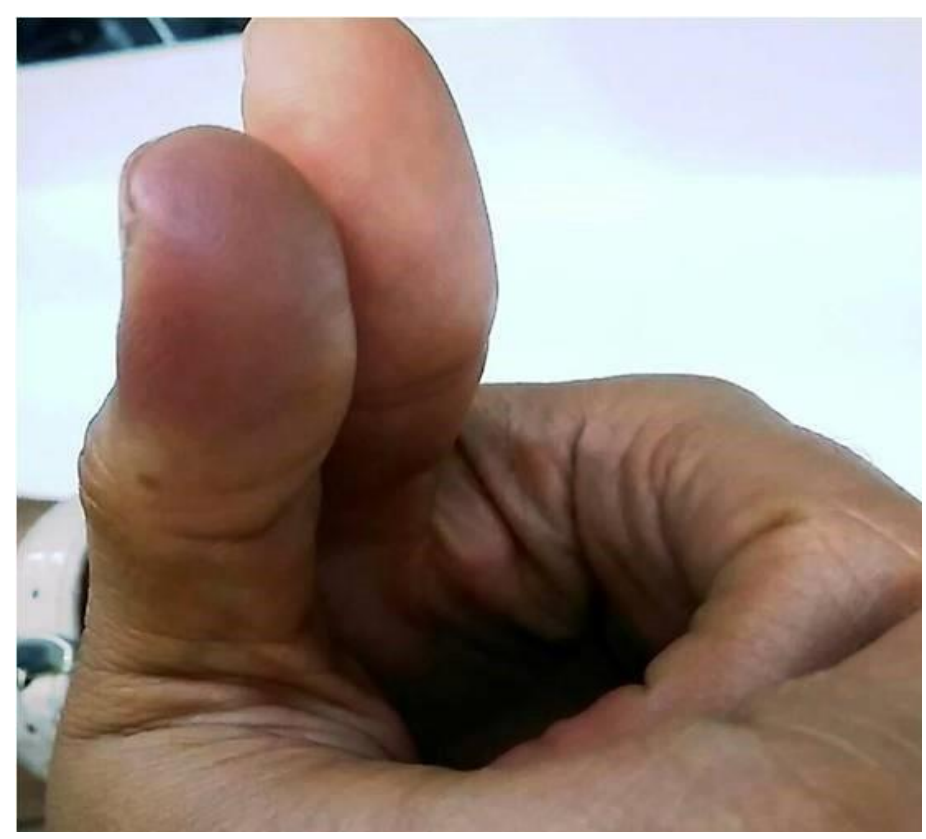

Figure 1. Clinical appearance violaceous color of the blue finger in our case.

The acute idiopathic blue finger is a benign and rare condition. It is characterized by an acute bluish discoloration of fingers which may be accompanied by pain $^{4}$. The acute blue finger appears to occur in all age groups, predominately affecting the female, middle-aged population ${ }^{1}$. Spontaneous acute blue finger syndrome is an entity different from ischemia or vasospasm. The mechanism of subcutaneous bruising has been offered. Lesions usually disappear without the stages of ecchymosis resorption. The patients presenting with acute blue fingers should be rapidly clinically evaluated. The absence of any thromboembolic disorders or systemic diseases indicates a benign clinical course ${ }^{2}$. In a prospective follow-up of 22 patients who had presented with sudden onset blue discoloration of a finger with normal radial and ulnar pulses, it was concluded that acute blue finger is generally a benign condition not suggestive of arterial embolization and with no threat from digit loss ${ }^{1}$.
Acute blue finger cases rarely refer to dermatology outpatient clinics. We want to emphasize that acute blue finger is usually benign disorder. However, it should be necessarily ruled out its possible causes.

\section{REFERENCES}

1. Cowen R, Richards T, Dharmadasa A, Handa A, Perkins JM. The acute blue finger: management and outcome. Ann R Coll Surg Engl 2008; 90: 557-60.

2. Weinberg I, Jaff MR. Spontaneous blue finger syndrome: A benign process. Am J Med 2012; 125: e1-e2.

3. Faraq M, Elmasry M, Mabote T, Elsayed A, Sunthareswaran R. Acute blue finger: a diagnostic challenge. BMJ Case Rep 2014; 15 : 2014. doi: 10.1136/bcr-2013-200290.

4. Manappallil RG, Jayaraj J. Blue finger syndrome: An unusual presentation of rheumatoid arthritis. J Clin Diagn Res 2017; 11: OD06-OD07. doi: 10.7860/ JCDR/ 2017/ 25300.9784. 